\title{
Coupled Vehicle Design and Network Flow Optimization for Air Transportation Systems
}

\author{
Christine Taylor* and Olivier L. de Weck \\ Massachusetts Institute of Technology, Cambridge, Massachusetts 02139
}

DOI: $\underline{10.2514 / 1.27320}$

\begin{abstract}
Traditionally, the design of a transportation system has focused on either vehicle design or the network flow, assuming the other as given. However, to define a system-level architecture for a transportation system, it is advantageous to expand the system boundary during the design process to include the network routing, the vehicle specifications, and the operations that couple the vehicle(s) and the network. In this paper, the transportation architecture is decomposed into these fundamental subsystems by classifying the decisions required to define each subsystem. Using an integrated transportation system formulation, the design of the transportation architecture can be obtained by concurrently optimizing the vehicle design and network flow. This is accomplished by embedding a linear programming solver in the perturbation step of simulated annealing to solve for the large number of linear constraints imposed by the capacity and demand requirements of the network. The benefits of this new formulation are illustrated through an example of an air transportation system for an overnight package delivery network in which a $10 \%$ improvement in cost is obtained over traditional network flow optimization. The improvement in system cost obtained can be attributed to the reduction in operational inefficiencies for the transportation system.
\end{abstract}

\section{Nomenclature}

\begin{tabular}{|c|c|}
\hline$A$ & $=$ aircraft type \\
\hline$C$ & $=$ cargo capacity, $\mathrm{lb}$ \\
\hline$f$ & $=$ fixed cost of allocating the aircraft, $\$ /$ day \\
\hline$(i, k)$ & $\begin{array}{l}=\text { aircraft route that starts at node } i \text { travels to node } k \text { and } \\
\text { returns to node } i\end{array}$ \\
\hline$(i, j, k)$ & $\begin{array}{l}=\text { package route that starts at node } i \text { travels through } \\
\text { node } k \text { and terminates at node } j\end{array}$ \\
\hline$m$ & variable cost of using the aircraft, $\$ / \mathrm{h}$ \\
\hline$N$ & number of cities \\
\hline$N_{\text {eng }}$ & number of engines \\
\hline$n_{i k}$ & number of aircraft on route $(i, k)$ \\
\hline$P_{i j}$ & $\begin{array}{l}=\text { package demand between cities } i \text { and } j \text { in a 24-h } \\
\text { period }\end{array}$ \\
\hline$R$ & $=$ range, $\mathrm{n}$ mile \\
\hline$T / W$ & $=$ thrust-to-weight ratio \\
\hline$V_{c}$ & $=$ cruise velocity, $\mathrm{kt}$ \\
\hline$W / S$ & $=$ wing loading, $\mathrm{lb} / \mathrm{ft}^{2}$ \\
\hline$x_{i j k}$ & number of packages on route $(i, j, k)$ \\
\hline
\end{tabular}

\section{Introduction}

$\mathbf{T}$ HE system-of-systems philosophy [1-3] has expanded the system boundary to encompass an integrated view of a system during the design process. Systems of systems are collections of systems (such as air vehicles) that can operate independently, but deliver more value when designed and operated as a coordinated ensemble. As we expand the definition of the system under consideration, we effectively enlarge the design control volume,

Presented as Paper 2202 at the 1st AIAA Multidisciplinary Design Optimization Specialist Conference, Austin, TX, 18-21 April 2005; received 16 August 2006; revision received 16 February 2007; accepted for publication 3 June 2007. Copyright (C 2007 by Christine Taylor and Olivier de Weck. Published by the American Institute of Aeronautics and Astronautics, Inc., with permission. Copies of this paper may be made for personal or internal use, on condition that the copier pay the $\$ 10.00$ per-copy fee to the Copyright Clearance Center, Inc., 222 Rosewood Drive, Danvers, MA 01923; include the code 0021-8669/07 \$10.00 in correspondence with the CCC.

*Postdoctoral Associate, Department of Aeronautics and Astronautics, 77 Massachusetts Avenue, Room 33-409; c_taylor@mit.edu. Student Member AIAA.

†ssociate Professor, Department of Aeronautics and Astronautics, Engineering Systems Division, 77 Massachusetts Avenue, Room 33-412; deweck@mit.edu. Associate Fellow AIAA. which defines the boundary of inputs and outputs of the system. The interior of the control volume is the design space under consideration, in which the designer can manipulate the components to achieve desired outputs, given the inherent physical constraints and the external constraints across the boundary. As the control volume expands, greater flexibility in decisions is achieved, but with this flexibility comes an increase in problem size and complexity.

Figure 1 depicts both an aircraft with subsystem components and an air transportation network that uses this aircraft. The control volume for vehicle design can be limited to any single subsystem, a limited interaction of subsystems, or the entire vehicle design. Similarly, network optimization theory limits the control volume to encompass only the transportation network, with the vehicle designs as given inputs to the problem. Aircraft designers assume that network demand and routing are given and produce a vehicle design that satisfies the operational requirements: namely, the range and capacity [4]. Operations researchers, on the other hand, often assume that vehicle specifications are known and held constant and seek to determine the best allocation of the fleet [5]. In reality, any vehicle is always a compromise design for its intended operations. Although the literature in each of the two areas (aircraft design and network flow optimization) taken separately is voluminous, previous work associated with the intersection between vehicle design and network operations is surprisingly sparse. This paper therefore focuses on the concurrent optimization of the aircraft design and network flow, assuming that such design freedom exists, effectively enlarging the control volume to include the aspects of Fig. 1 .

Maier [1] defined a system of systems by the level of managerial and operational independence of the components of the system. In air transportation networks, multiple aircraft, each of which can be operated and managed independently, collaborate to satisfy multiple independent demands. By analyzing the design of an aircraft in the context of a transportation network, the coupling between the aircraft design and network flow can be investigated and exploited to provide more efficient operations overall.

Traditionally, optimization of the flow in a transportation network focuses on determining the optimal set of operations for a given vehicle or set of vehicles such that the prescribed demand is satisfied (Ahuja et al. ). Investigations into the vehicle routing problem (VRP) began with Dantzig and Ramser [7] with model development and a discussion of the computational approaches to obtain solutions for the truck routing problem. Toth and Vigo [8] presented a detailed examination of the VRP and its variants. Simchi-Levi et al. [9] used transportation network modeling to solve a school bus routing 


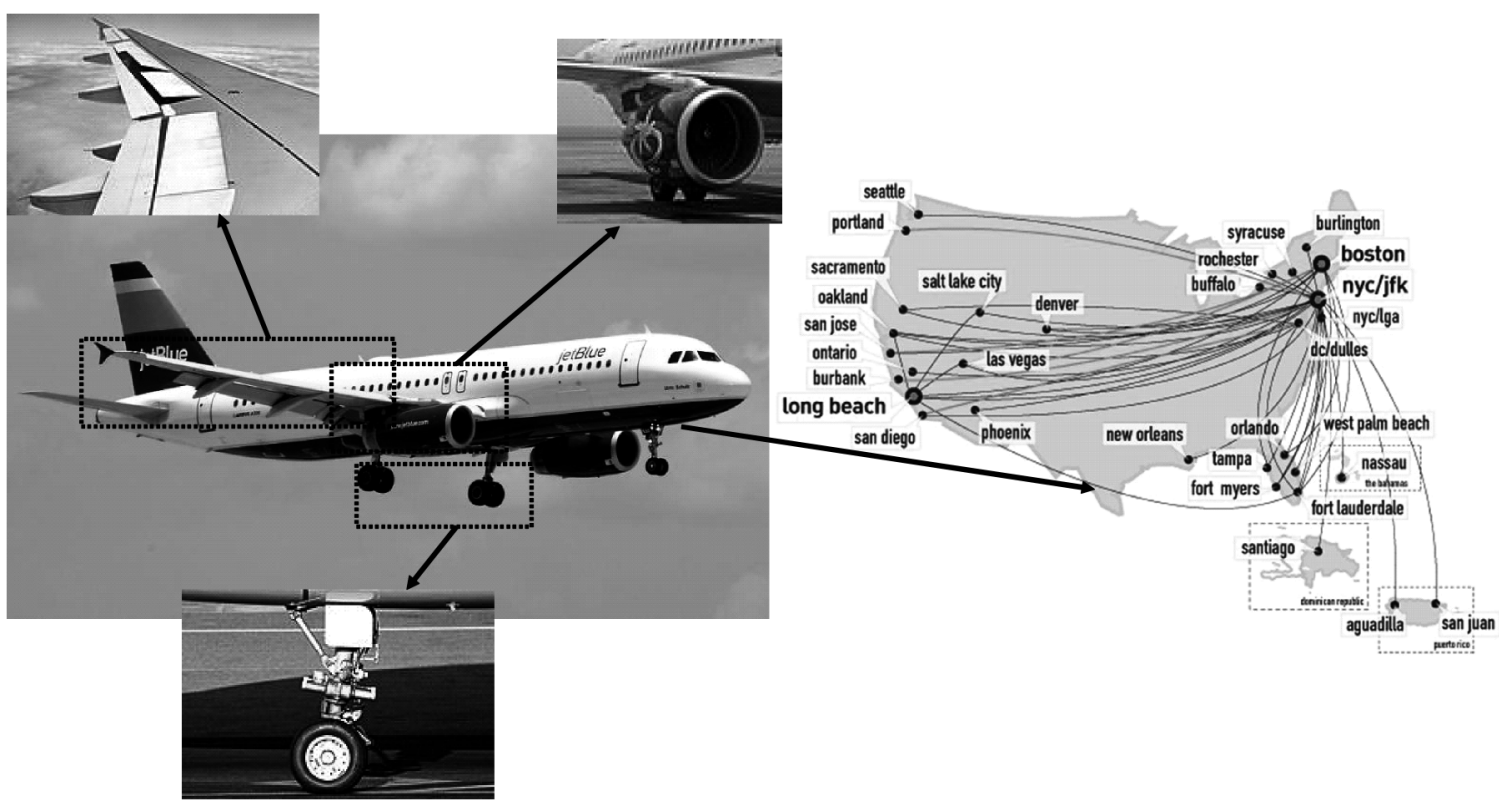

Fig. 1 Aircraft A320 with specific subsystems defined in greater detail as inserts (left) and Jet Blue air transportation network (right).

problem, in which the primary constraints focused on the timing restrictions inherent in school bus pickups and dropoffs. Using transportation networks, they were able to find an optimal allocation of vehicles to routes and schedules.

For air transportation systems, the vehicle routing problem is modified slightly to incorporate the additional constraints inherent in flight. Yang and Kornfeld [10] considered the optimal allocation of a set of predefined vehicles for an overnight package delivery system, in which the objective is to minimize the total cost for a single day of operation. Barnhart et al. [5] combined the traditional routing problem with the fleet assignment problem to develop a more robust methodology for defining the flight scheduling for an airline.

Recently, investigations into the design of vehicles to fulfill multiple operations have been considered to understand the impact of these requirements on the vehicle design characteristics. Frommer and Crossley [11] compared the designs of fixed geometry and morphing geometry aircraft operating as a fleet, for satisfying multiple predefined operational scenarios. Crossley et al. [2], specified the operations (namely, the routes), and the objective was to define a vehicle design that satisfies the prescribed demand at the lowest cost for a day of operation. This work was extended by Mane et al. [3] when the problem size under consideration was increased significantly, which showed the scalability of the approach to larger problems.

In the context of space operations, Meissinger and Collins [12] examined the design of a single multifunction orbit transfer vehicle (OTV) that not only fulfills the current mission requirements but has the flexibility to be extended for potential future mission objectives. Given the new space exploration initiative, an increasing number of investigations into the design of a space exploration system that will travel to both the moon and Mars have been conducted. In Wooster et al. [13] and Stanley et al. [14], multiple predefined reference missions are used to evaluate the design of a space transportation system for exploration.

By expanding the system boundary to include the transportation network flow and operations into the vehicle design, operational inefficiencies can be reduced, but at the cost of increased computational complexity. The resulting model has both the mixedinteger linear constraints inherent in network flow and routing problems, as well as the nonlinear analysis functions required to analyze aircraft or spacecraft designs. As such, it is necessary to find efficient solution methods to solve problems of this structure to obtain good solutions in a reasonable time period.

This paper investigates the benefits of solving a concurrent aircraft design and network flow problem. In Sec. II, a decomposition of the air transportation system is presented. Section III explains the optimization approach used to solve the combined (integrated) transportation optimization problem. Section IV presents the air transportation example analyzed in this paper and compares the results obtained through the traditional design approaches for separate network and vehicle optimization with the concurrent optimization approach presented in this paper. Section $\mathrm{V}$ compares and analyzes the results obtained from the three approaches. Section VI reviews the contributions and results presented and discusses continuing work on this topic.

\section{Problem Formulation}

The integrated transportation system design problem consists of four components: the network flow, the vehicle design, the operations constraints, and the system-level objective. As shown in Fig. 2, the vehicle and the network are the subsystems that determine the cost of the transportation system, and the operations define the constraints that couple them. The following subsections describe the models and assumptions required to define each component of the problem.

\section{A. Network Model Formulation}

The network subsystem defines the allocation of vehicles and cargo (e.g., packages) to routes through the network, whereby the network is defined by pairs of cities that are to be linked. In our

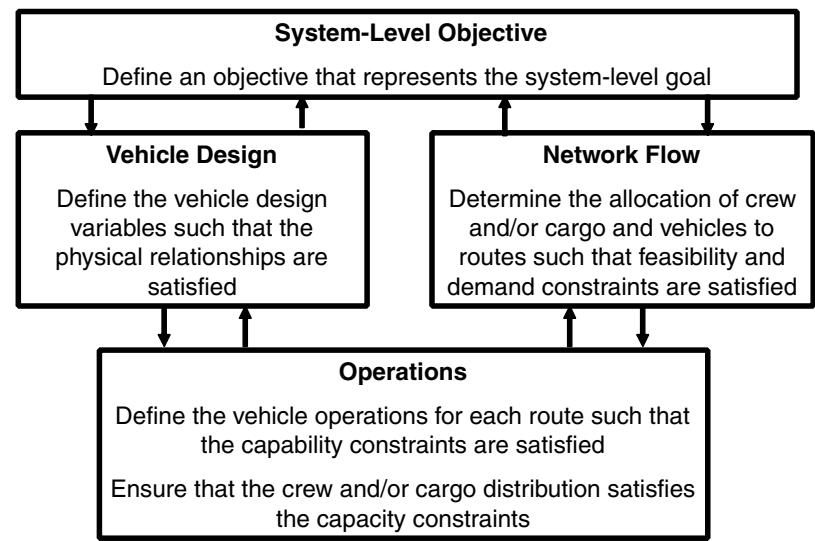

Fig. 2 Diagram of the integrated transportation system model. 
model, an aircraft flies between two cities and performs the roundtrip flight once in a 24-h period. The number of aircraft of type $A$ flying from city $i$ to city $k$ and returning is defined as $n_{i k}^{A}$. Because only feasible routes are defined, the vehicle allocation constraints simply impose a limit on the number of aircraft of a given type flying a given route.

$$
n_{i k}^{A} \leq 10 \quad k=1, \ldots, N \quad \forall A
$$

The (cargo) package flow constraints ensure that the demand of each city pair is fulfilled. Although aircraft can fly only round trips between two cities, we assume that packages may travel through an additional city toward the destination. By defining a route $(i, j, k)$ as starting at city $i$ traveling through city $k$ and terminating at city $j$, the number of packages traveling this route can be defined as $x_{i j k}$.

The demand constraints that govern the feasibility of the package flow are supplied in Eq. (2).

$$
\sum_{k=1}^{N} x_{i j k}=P_{i j} \quad i, j=1, \ldots, N
$$

where $P_{i j}$ is the package demand from city $i$ to city $j$, and $N$ is the total number of cities in the network.

Figure 3 defines both the aircraft and package variables in the context of a simple three-city-network example. On the left, the first leg shows the outbound flights and the first segment of the trip for packages. On the right, the second leg shows the return flights and the final segment of the package routes. As shown in Fig. 3, packages can be delivered directly on the first leg $x_{i j j}$, wait to be delivered on the second leg $x_{i j i}$, or travel both legs toward the destination $x_{i j k}$.

\section{B. Vehicle Model Formulation}

The vehicle subsystem determines the architectural and performance characteristics of the aircraft design. The design of an aircraft of type $A$ is defined by the range $R^{A}$, capacity $C^{A}$, cruise velocity $V_{c}^{A}$, wing loading $W^{A} / S$, thrust-to-weight ratio $T^{A} / W$, and number of engines $N_{\mathrm{eng}}^{A}$. Equation (3) defines the range of feasible values for each of the design variables.

$$
1000 \mathrm{n} \text { mile } \leq R^{A} \leq 5000 \mathrm{n} \text { mile }
$$

$$
\begin{gathered}
5000 \mathrm{lb} \leq C^{A} \leq 250,000 \mathrm{lb} \quad 250 \mathrm{kt} \leq V_{c}^{A} \leq 550 \mathrm{kt} \\
95 \frac{\mathrm{lb}}{\mathrm{ft}^{2}} \leq \frac{W^{A}}{S} \leq 150 \frac{\mathrm{lb}}{\mathrm{ft}^{2}} \quad 0.3 \leq \frac{T^{A}}{W} \leq 0.4 \\
N_{\mathrm{eng}}^{A} \in\{1,2,3,4\}
\end{gathered}
$$

Additionally, a constraint on takeoff length is included to ensure that aircraft can fly out of any major airport.

$$
d_{\mathrm{TO}}=\frac{1.21\left(W^{A} / S\right)}{g \rho C_{L}\left(T^{A} / W\right)} \leq 9000 \mathrm{ft} \quad \forall A
$$

where $\rho$ is the density at sea level, $g$ is the gravitational constant, $C_{L}$ is the lift coefficient at takeoff, and the factor of 1.21 is a constant to account for differences in aircraft performance during takeoff, as recommended by Anderson [15].
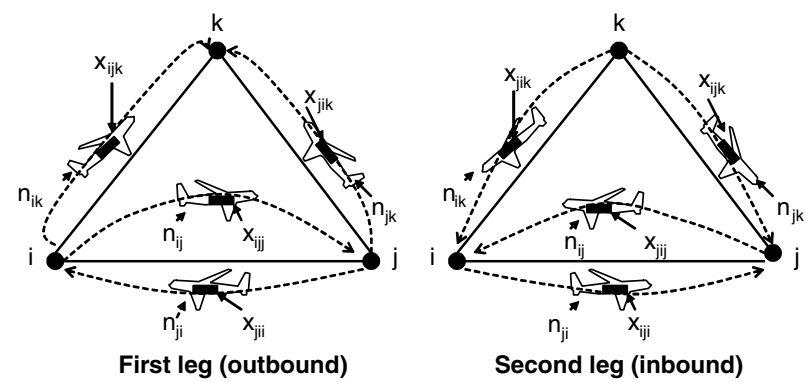

Fig. 3 Description of network flow variables.

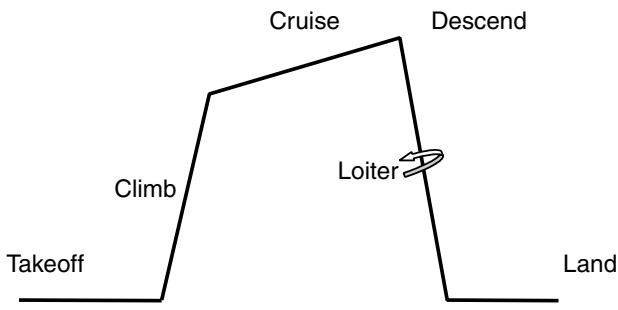

Fig. 4 Diagram of a simple cruise profile.

The takeoff weight of the aircraft can be calculated from the design variables assuming a simple cruise profile, as shown in Fig. 4. Although the takeoff weight is not constrained explicitly, it is a required input to the cost function described in the next section. Using a model provided by Raymer [4], a weight ratio is assigned to each segment of the cruise profile. The weight ratios for takeoff, climb, and decent/landing are typical values provided by Raymer and are listed in Table 1 .

The weight ratios for the cruise $W_{C}$ and loiter $W_{L}$ segments are taken from the Breguet range and endurance equations, respectively, and are listed in Eq. (5).

$$
W_{C}^{A}=\exp \frac{-R^{A} S_{\mathrm{FC}}}{V_{c}^{A} L / D} \quad W_{L}^{A}=\exp \frac{-t S_{\mathrm{FC}}}{L / D}
$$

where $S_{\mathrm{FC}}$ is the specific fuel consumption of the aircraft, $L / D$ is the lift-to-drag ratio, and $t$ is the time spent loitering before landing. The nominal values of these parameters are listed in Table 2. By multiplying the weight ratios together, the total weight ratio $\bar{W}_{T}^{A}$ for the entire flight profile of vehicle $A$ can be estimated. The fuel fraction $f_{f}^{A}$ of aircraft $A$ is computed from the total weight ratio, as shown in Eq. (6), whereby a $6 \%$ fuel reserve is assumed.

$$
f_{f}^{A}=1.06\left(1-W_{T}^{A}\right)
$$

The total takeoff weight $W_{0}^{A}$ is defined to be the sum of the cargo weight, the weight of the fuel for a fully loaded tank, and the structural weight of aircraft $A$. Rearranging this relationship, we can express the total takeoff weight of the aircraft as shown in Eq. (7).

$$
W_{0}^{A}=\frac{W_{p}^{A}}{1-f_{f}^{A}-s_{f}^{A}}
$$

where the structural fraction $s_{f}^{A}$ is the ratio of the structural mass to the total takeoff mass. The payload weight $W_{p}^{A}$ is the total cargo mass of the aircraft plus the weight of two crew members, because we focus on cargo flights in this paper. The cargo mass is assumed to be equal to the aircraft capacity $C^{A}$, which decouples the aircraft performance constraints (e.g., on range) from the package distribution. The structural or dry weight of the aircraft accounts for the total unloaded and unfueled aircraft weight and is estimated by an empirically

Table 1 Defined weight ratios for simple cruise profile segments

\begin{tabular}{lc}
\hline \hline Segment & Weight ratio \\
\hline Takeoff & 0.97 \\
Climb & 0.985 \\
Descent/landing & 0.995 \\
\hline \hline
\end{tabular}

Table 2 Parameter values for aircraft design

\begin{tabular}{lc}
\hline \hline Parameter & Value \\
\hline$S_{\mathrm{FC}}, 1 / \mathrm{s}$ & 0.6 \\
$\mathrm{~L} / \mathrm{D}$ & 17 \\
$t, \mathrm{~min}$ & 30 \\
\hline
\end{tabular}


derived formula for vehicle mass, taken from Raymer [4] and shown in Eq. (ㅁ).

$$
s_{f}^{A}=1.02 W_{0}^{A-.06}
$$

The total aircraft weight and the weight of the fuel are determined by numerically solving the system of equations defined by Eqs. (7) and ().

\section{Operations Model Formulation}

The operations of a transportation system determine how the vehicle performs on a given route and is defined by two sets of equations: capability and capacity constraints. The capability constraints, given in Eq. (9), require that a given vehicle cannot travel between two cities for which the distance is greater than the range of the aircraft (assuming that refueling is not allowed).

$$
d_{i k} \leq R^{A} \quad k=1, \ldots, N \quad \forall A
$$

To formulate the capacity constraints, we first define the capacity of route $(i, k)$ as $G_{i k}$, as in Eq. (10), and then the capacity constraints can be formulated as shown in $\overline{\mathrm{Eq}}$. (11).

$$
G_{i k}=\sum_{A} n_{i k}^{A} C^{A}
$$

$$
\begin{aligned}
& \sum_{j=1}^{N} x_{i j k} \leq G_{i k} \quad i, k=1, \ldots, N \\
& \sum_{i=1}^{N} x_{i j k} \leq G_{j k} \quad j, k=1, \ldots, N
\end{aligned}
$$

Because we assume that a given vehicle travels only between two cities, the capacity of a route is the same on the return leg as it is on the outbound leg.

\section{System Objective}

In this paper, the objective is to minimize the total system cost for a single day of operation. Each aircraft has two associated cost values: a fixed cost that is associated with an aircraft's allocation, and a variable cost that is associated with an aircraft's operation. The aircraft's performance parameters define both the fixed and variable costs for the design, which are taken from the development and procurement costs of aircraft (DAPCA 4) models provided by Raymer [4].

The cost model uses the structural weight of the aircraft $W_{s}$, velocity $V_{c}$, number of engines $N_{\text {eng }}$, and thrust per engine $T_{\text {eng }}$ as inputs to compute the research, development, testing, and evaluation costs. These nonrecurring costs are used to determine the depreciation of the aircraft. The fixed cost $f^{A}$ of the aircraft is the cost per day of ownership of aircraft $A$ and is equivalent to the perday depreciation of the aircraft. The variable costs $m^{A}$ are the recurring costs associated with the usage of aircraft $A$ and can therefore be computed as the cost per hour of aircraft flight.

The total system operating costs are therefore defined as

$$
J=\sum_{i=1}^{N} \sum_{j=1}^{N} \sum_{A} c_{i k}^{A} n_{i k}^{A}
$$

where $c_{i k}^{A}$ represents the cost of aircraft $A$ traveling on route $(i, k)$, as expressed in Eq. (13).

$$
c_{i k}^{A}= \begin{cases}f^{A}+m^{A} \frac{2 d_{i k}}{V_{c}^{A}}, r^{A} \geq d_{i k}, & i \neq k \\ \infty, r^{A}<d_{i k}, & i \neq k \\ 0, & i=k\end{cases}
$$

Equation (13) imposes a cost equal to the fixed cost plus twice the time required to travel a single leg of the trip (to account for the round trip) multiplied by the variable cost per hour of flying the aircraft, if the aircraft can fly a given leg, as determined by the range requirement. If an aircraft does not have the range required to travel a given leg, a very large cost is assigned to prohibit the selection. Finally, in the current model, stopover and storage at a given city is free (for cargo), and therefore same-city transfers have no cost associated with them.

\section{Concurrent Design Optimization Methodology}

The integrated transportation system design problem, as described earlier, assimilates all of the preceding defined variables and constraints into a single system-level problem. As such, the design problem can be classified as a mixed-integer nonlinear programming problem (MINLP), which is difficult to solve effectively (Bertsekas [16] and Bertsimas and Weismantel [17]). Typically, either simplifications are made to the constraints or the problem is decomposed using methods such as collaborative optimization (Braun and Kroo [18]) or bilevel integrated system synthesis (BLISS) (Sobieszczanski-Sobieski [19]). However, due to the special structure of the integrated transportation system design problem, a different type of decomposition was developed to effectively solve the problem.

Heuristic optimization algorithms are often employed to solve MINLPs because they can handle problems of any mathematical structure. One such heuristic optimization algorithm that provides a useful approach to design space exploration is simulated annealing (SA) [20]. SA can solve problems with mixed-integer variables and nonlinear constraints and analysis functions by perturbing the design vector and evaluating the likelihood of improving the current objective function value by moving to a new point in the design space. In addition, SA has the added property that the acceptance of new design points changes as the algorithm evolves and thus mimics gradient-based decisions near the end of the optimization problems with many constraints can be difficult to formulate in this framework.

For highly constrained optimization problems, it is often desirable to continuously perturb the design variables until a feasible design vector is selected. However, for problems with continuous variables and equality constraints, it is unlikely that random perturbations of the design variables will produce feasible solutions. For this reason, it is desirable to use methods for constraint solving that can determine a new feasible set of design points. By embedding a linear programming (LP) solver within SA, the difficulties with satisfying many linear constraints within the heuristic optimizer are ameliorated. This embedded optimization implementation is what enables an efficient solution of the coupled vehicle design and network flow problem. Using this approach, a single system-level design problem can be formulated using a heuristic optimization algorithm to navigate the design space, and the linear constraints governing the network flows and capacity constraints are computed by the embedded LP solver to ensure a feasible package flow.

Table 3 shows the dependence of the constraints and objective function described in Sec. II on the variables in the problem. Here, $x_{\text {veh }}$ represents the vehicle design variables presented in Eq. (3), $n_{i k}$

Table 3 Decomposition of integrated air transportation system design problem

\begin{tabular}{lccc}
\hline \hline & \multicolumn{4}{c}{ Variables } \\
\hline Equation & $x_{\text {veh }}$ & $n_{i k}$ & $x_{i j k}$ \\
N-max [Eq. (1)] & & $\mathrm{X}$ & \\
Demand [Eq. (2)] & & & $\mathrm{X}$ \\
Takeoff [Eq. (7)] & $\mathrm{X}$ & & \\
Range [Eq. (9)] & $\mathrm{X}$ & $\mathrm{X}$ & \\
Capacity [Eq. (11)] & $\mathrm{X}$ & $\mathrm{X}$ & $\mathrm{X}$ \\
Cost [Eq. (12)] & $\mathrm{X}$ & $\mathrm{X}$ & \\
\hline \hline
\end{tabular}

Data available online at http://ocw.mit.edu/OcwWeb/Aeronautics-andAstronautics/16-888Spring-2004/CourseHome/index.htm. 


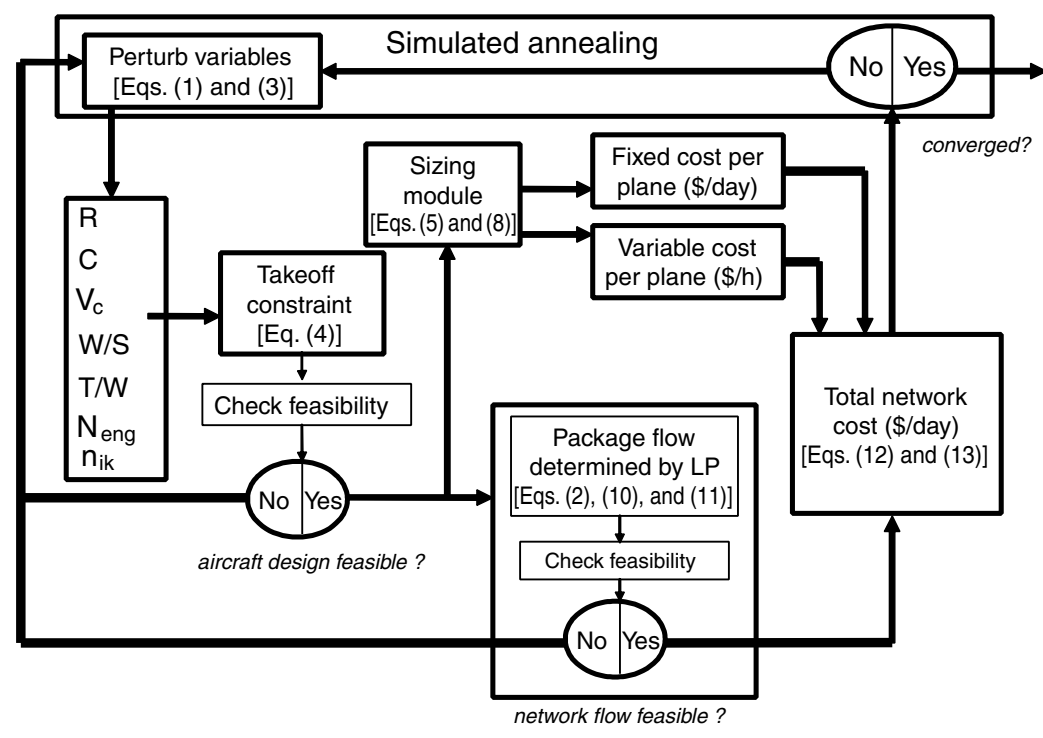

Fig. 5 Integrated transportation system design optimization with simulated annealing.

defines the number of aircraft on each route, and $x_{i j k}$ is the package flow on each route. Examining Table 3 reveals that the takeoff constraint is dependent only on the vehicle design variables $x_{\mathrm{veh}}$, and the maximum aircraft constraint depends solely on the vehicle routing variables $n_{i k}$. Therefore, these decisions can be made independent of any other information. Another important observation that can be obtained from examining Table $\underline{3}$ is that both the objective function and range constraint are independent of the package flow variables. This observation allows for the package flow variables to be defined as a secondary decision. The demand constraints and capacity constraints are the only two sets of constraints that involve the package flow variables. Thus, by defining both the vehicle design and routing variables before the evaluation of the demand and capacity constraints, a linear system of constraints governing only the package flow variables is defined, which can be used advantageously in the optimization.

Figure 5 presents the optimization flow diagram for the integrated transportation system design problem. The design vector consists of the aircraft design variables and the network allocation variables. By perturbing the values of these variables, the optimizer can evaluate the takeoff constraint and determine if the aircraft design is feasible, given this vehicle design constraint. Given a feasible aircraft design, the demand and capacity constraints are evaluated by an embedded LP solver that determines if a feasible network flow exists. If there a feasible solution exists, the current design vector is evaluated to determine the total system cost; otherwise, the design variables are perturbed to define a new design vector. This process continues until the algorithm converges.

\section{Example Air Transportation System}

To evaluate the effectiveness of the integrated transportation system design methodology against conventional practice, a case study of an air transportation system for overnight package delivery network, which was presented earlier by Yang and Kornfeld [10], is considered. The models described earlier are implemented for a network consisting of the seven largest cities, in terms of demand to and from Atlanta (ATL), Boston (BOS), Chicago (ORD), Dallas (DFW), Los Angeles (LAX), New York (JFK), and San Francisco (SFO). The distance and demand information is provided in Tables $\underline{4}$ and 5, respectively. The network is assumed to have symmetric demand between each city pair, and the network is fully connected (connections represent the straight-line distances between every city).

For the example network defined, traditional optimization approaches are employed to solve the problem to provide a basis for comparison for the integrated optimization methodology presented in this paper. The traditional optimization methodology embodies two views: network optimization with given vehicles and vehicle optimization with a given network. The following sections detail the results of these two cases, as well as the results of the concurrent optimization, using the framework developed in Fig. 5.

\section{A. Case 1: Network Optimization}

In traditional network optimization, a set of vehicles are defined, each with an associated cost and capability. Using these predefined vehicle parameters, an optimal allocation of vehicles to routes can be defined to meet the demand of the network. In Yang and Kornfeld [10], three types of aircraft are chosen to provide a representative sample for a small (plane A), medium (plane B), and large (plane C) airplane. Using the cost calculation described earlier, the fixed and variable costs can be calculated from the vehicle characteristics, and the relevant parameters of each aircraft are given in Table 6 .

Using the parameters listed in Table 6 and the network and cost models described in Sec. II, an optimal allocation of vehicles to routes can be determined by employing CPLEX as a linear mixedinteger optimization algorithm. For the example network defined in Tables 4 and 5, the minimum total cost for one day of operations is $\$ 517,0 \overline{30}$, and the optimal allocation of aircraft to routes is depicted in Fig. 6 .

By examining Fig. 6 and Table 7, it is shown that only the medium (plane B) and large (plane C) airplanes are allocated, due to both the range and capacity constraints. The solution defines Chicago as a hub and additionally routes incoming flights from every city except San Francisco into Dallas. Because San Francisco has only two outgoing flights, it is necessary to use the largest-capacity aircraft on both routes to accommodate the packages. Plane $\mathrm{C}$ is also allocated on the New York to Los Angeles and New York to Chicago routes, to accommodate the large demand originating in New York.

\section{B. Case 2: Vehicle Optimization}

In traditional vehicle optimization, the network flow is defined a priori and the vehicle design characteristics are optimized to produce

Table 4 City-to-city distances for example air transportation network, $n$ mile

\begin{tabular}{lrrrrrrr}
\hline \hline & ATL & BOS & ORD & DFW & LAX & JFK & SFO \\
\hline ATL & 0 & 934 & 622 & 688 & 1921 & 756 & 2179 \\
BOS & 934 & 0 & 882 & 1538 & 2629 & 183 & 2729 \\
ORD & 622 & 882 & 0 & 806 & 1767 & 713 & 1866 \\
DFW & 688 & 1538 & 806 & 0 & 1257 & 1360 & 1518 \\
LAX & 1921 & 2629 & 1767 & 1257 & 0 & 2454 & 330 \\
JFK & 756 & 183 & 713 & 1360 & 2454 & 0 & 2560 \\
SFO & 2179 & 2729 & 1866 & 1518 & 330 & 2560 & 0 \\
\hline \hline
\end{tabular}


Table 5 Daily demand for example air transportation network, lb

\begin{tabular}{lrrrrrrr}
\hline \hline & ATL & BOS & ORD & DFW & LAX & JFK & SFO \\
\hline ATL & 0 & 14,045 & 31,313 & 19,984 & 34,506 & 57,949 & 37,318 \\
BOS & 14,045 & 0 & 27,261 & 17,398 & 30,041 & 50,451 & 32,489 \\
ORD & 31,313 & 27,261 & 0 & 38,788 & 66,975 & 112,479 & 72,434 \\
DFW & 19,984 & 17,398 & 38,788 & 0 & 42,743 & 71,784 & 46,227 \\
LAX & 34,506 & 30,041 & 66,975 & 42,743 & 0 & 123,948 & 79,820 \\
JFK & 57,949 & 50,451 & 112,479 & 71,784 & 123,948 & 0 & 134,050 \\
SFO & 37,318 & 32,489 & 72,434 & 46,227 & 79,820 & 134,050 & 0 \\
\hline \hline
\end{tabular}

Table 6 Predefined aircraft specifications for

\begin{tabular}{lcrr}
\multicolumn{4}{c}{ case 1 } \\
\hline \hline Parameter & Plane A & Plane B & Plane C \\
\hline Capacity $C, \mathrm{lb}$ & 5000 & 72,210 & 202,100 \\
Range $R, \mathrm{n}$ mile & 1063 & 3000 & 3950 \\
Velocity $V_{c}, \mathrm{kt}$ & 252 & 465 & 526 \\
Fixed cost $f, \$ /$ day & 1481 & 10,616 & 26,129 \\
Linear cost $m, \$ / \mathrm{h}$ & 758 & 3116 & 7194 \\
\hline \hline
\end{tabular}

the lowest system cost. For the traditional vehicle design optimization problem, a hub-spoke network configuration is assumed, in which a single city in the network is designated as the hub and all routes in the network connect to this city. More precisely, a hub is defined as a node with nodal degree $N-1$, where $N$ represents the number of nodes in the network. The optimal vehicle design characteristics defined are based on the best compromise in performance for the network configuration. The vehicle optimization requires an algorithm that can accommodate the mixed-integer variables and nonlinear analysis functions required to define the vehicle design and allocation. As such, simulated annealing (Kirkpatrick et al. [20]) is chosen as the optimization algorithm for this case.

The optimal cost for this hub-spoke network and optimized cargo aircraft design is $\$ 570,720$ per day, and the design parameters are provided in Table $\underline{8}$ for the corresponding network configuration shown in Fig. 7 and detailed in Table 9.

If we examine Table $\underline{8}$ and Fig. 7, we see that the range of the aircraft designed is between that of planes A and B from case 1, because a full $\approx 2500 \mathrm{n}$ mile transcontinental flight is not required, due to the hub at ORD. The capacity of the aircraft designed by the vehicle optimization is between that of aircraft $\mathrm{B}$ and $\mathrm{C}$, and it reflects the large demand requirements for direct flights into Chicago. Although the vehicle is designed to reduce inefficiencies in the network, the requirement of only using direct flights (forcing a hub at ORD) and only allowing one single type of aircraft actually increases the system cost by $\approx 10 \%$ relative to case 1 .

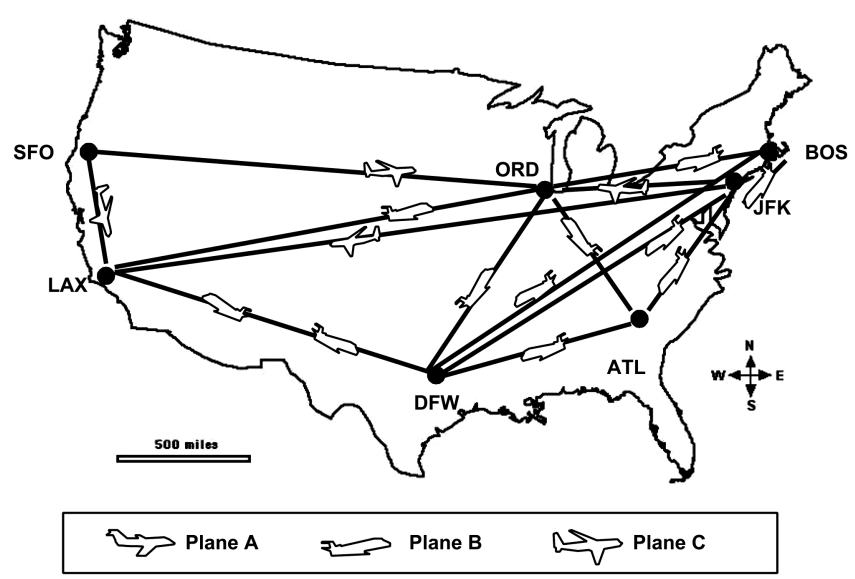

Fig. 6 Optimal configuration for case 1.
Table 7 Routing matrix for case 1

\begin{tabular}{|c|c|c|c|c|c|c|c|}
\hline & ATL & BOS & ORD & DFW & LAX & JFK & SFO \\
\hline ATL & & & B & B & & & \\
\hline BOS & & & B & B & & B & \\
\hline ORD & & & & & & & \\
\hline DFW & & & B & & B & & \\
\hline LAX & & & B & B & & & \\
\hline JFK & B & & C & B & C & & \\
\hline SFO & & & C & & C & & \\
\hline
\end{tabular}

Table 8 Aircraft specifications for case 2

\begin{tabular}{lc}
\hline \hline Parameter & New plane design \\
\hline Capacity $C, \mathrm{lb}$ & 128,050 \\
Range $R, \mathrm{n}$ mile & 1920 \\
Velocity $V_{c}, \mathrm{kt}$ & 540 \\
Wing loading $W / S, \mathrm{lb} / \mathrm{ft}^{2}$ & 134 \\
Thrust to weight $T / W$ & .315 \\
Number of engines $N_{\text {eng }}$ & 2 \\
Fixed cost $f, \$ /$ day & 14,106 \\
Linear cost $m, \$ / \mathrm{h}$ & 4083 \\
\hline \hline
\end{tabular}

Table 9 Routing matrix for case 2

\begin{tabular}{|c|c|c|c|c|c|c|c|}
\hline & ATL & BOS & ORD & DFW & LAX & JFK & SFO \\
\hline ATL & & & 2 & & & & \\
\hline BOS & & & 2 & & & & \\
\hline ORD & & & & & & & \\
\hline DFW & & & 2 & & & & \\
\hline LAX & & & 3 & & & & \\
\hline JFK & & & 5 & & & & \\
\hline SFO & & & 4 & & & & \\
\hline
\end{tabular}

\section{Case 3: Concurrent Vehicle and Network Optimization}

For the integrated transportation network design, the vehicle, network, and operations definitions are concurrently optimized. The design vector includes variables that define both the vehicle design and network flow, and the system is subject to the constraints that govern the vehicle, network, and operations. The integrated transportation system design problem is solved using the methodology described in Sec. II.

If we consider the design of a single vehicle and concurrently optimize the vehicle characteristics and the flow through the network for the example air transportation network, the optimal system cost is $\$ 463,723$, which is a reduction in cost of $10 \%$ over the traditional network optimization and a reduction of $18 \%$ over the traditional vehicle optimization. The optimal vehicle design parameters for the concurrent design optimization are listed in Table 10, and the optimal configuration is provided in Fig. 8 and detailed in Table 11.

The concurrently optimized solution presented in Table 10 and Fig. $\underline{8}$ is sized to be slightly smaller than airplane B. The reduction in range would no longer accommodate transatlantic flights, but does 


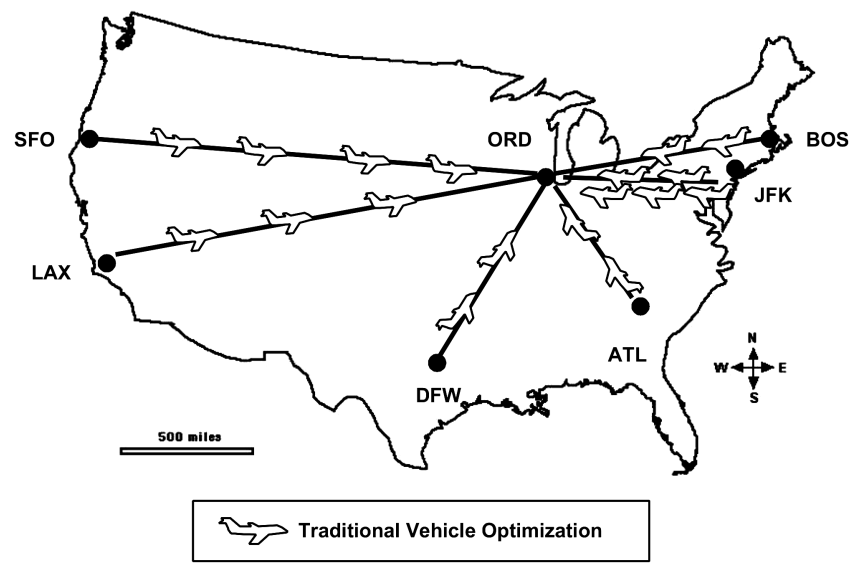

Fig. 7 Optimal configuration for case 2.

still satisfy the transcontinental distance requirements for the New York to Los Angeles and New York to San Francisco flights. By reducing the range and the capacity of the vehicle design slightly, a reduction in aircraft costs is obtained and it is now cheaper to use more of these aircraft. Again, because direct flights are not required (a strict hub-spoke network is not enforced) the capacity of the concurrently optimized aircraft is less than that of the vehicle optimization design from case 2 , but the resulting aircraft has a greater range.

\section{Discussion}

The integrated transportation system design methodology exploits the coupling of the vehicle and network by defining a more efficient set of operations for the transportation system. This effect can best be explained and visualized by plotting the distance vs demand of each city pair in the network (Fig. 9). In addition, vehicle design points are included by plotting the range vs capacity of the aircraft involved in cases $1-3$. The relationship of the vehicle design specifications to the network requirements can be interpreted as follows. All demand points lying within the (dashed) bounding box of a vehicle design point can be fulfilled by a single direct flight of that vehicle. Any points to the right of the vehicle design point but below the upper bound of the box require at least one connection (stopover or hop), because the distance exceeds the aircraft's range. Additionally, any points above the vehicle design point but left of the right bound of the box require more than one flight, because the demand exceeds the capacity of a single vehicle.

Table 10 Aircraft specifications for case 3

\begin{tabular}{lc}
\hline \hline Parameter & New plane design \\
\hline Capacity $C, \mathrm{lb}$ & 69,884 \\
Range $R, \mathrm{n}$ mile & 2560 \\
Velocity $V_{c}, \mathrm{kt}$ & 550 \\
Wing loading $W / S, \mathrm{lb} / \mathrm{ft}^{2}$ & 106 \\
Thrust to weight $T / W$ & .302 \\
Number of engines $N_{\text {eng }}$ & 2 \\
Fixed cost $f, \$ /$ day & 9633 \\
Linear cost $m, \$ / \mathrm{h}$ & 2807 \\
\hline \hline
\end{tabular}

Table 11 Routing matrix for case 3

\begin{tabular}{|c|c|c|c|c|c|c|c|}
\hline & ATL & BOS & ORD & DFW & LAX & JFK & SFO \\
\hline ATL & & & 1 & 1 & & 1 & \\
\hline BOS & & & 1 & & & 2 & \\
\hline ORD & & & & & & 1 & 1 \\
\hline DFW & & & 1 & & 1 & & \\
\hline LAX & & & 1 & 1 & & 2 & 1 \\
\hline JFK & & & 1 & 1 & & & 1 \\
\hline SFO & & & 1 & & 1 & 1 & \\
\hline
\end{tabular}

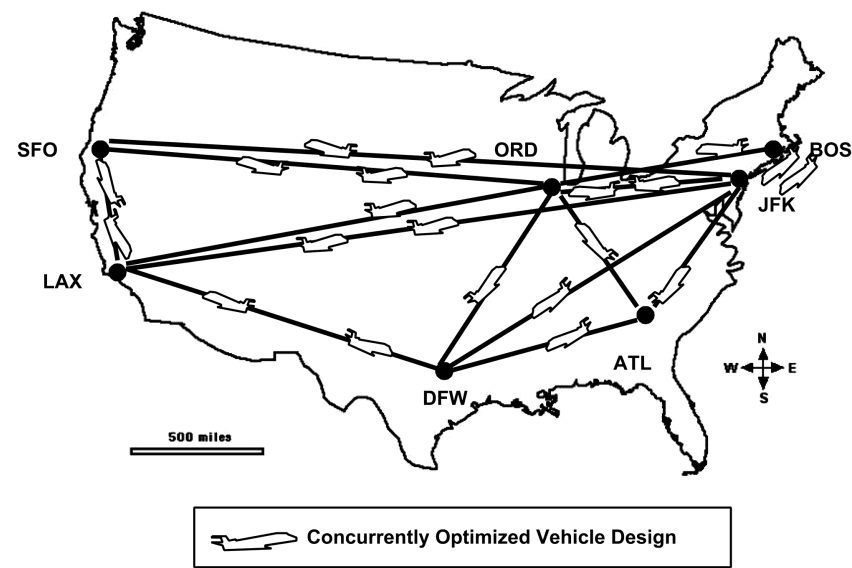

Fig. 8 Optimal configuration for case 3.

Figure 9 displays the distance and demand of the seven-city network and the vehicle design points from all three cases, as summarized in Table 12. By examining Fig. 9, we see that the integrated optimized aircraft design (case 3 ) has a range that can just handle the distance requirements of New York to Los Angeles and New York to San Francisco flights (with $6 \%$ fuel margin), but the demand between these cities is almost twice the aircraft's capacity. If we examine Fig. 8, we see that there are two flights from Los Angeles to New York and a direct flight in each direction between New York and San Francisco, which can accommodate the New York to Los Angeles and New York to San Francisco demands, respectively. However, it is important to realize that some of the flow between these city pairs may be handled by other connecting flights, because there is a Boston to New York flight that may require some of the Boston to Los Angles and Boston to San Francisco packages to be flown on the return flights from Los Angeles and San Francisco, respectively. Thus, the optimal solution is a hybrid between a huband-spoke and direct architecture.

Examining Table 12 further reveals the effect of the design decisions on the overall unit cost of the aircraft. The unit cost is defined as the cost per pound of cargo shipped per nautical mile flown, assuming the aircraft is at full capacity and traveling the maximum range for a round-trip flight. The unit cost allows the different aircraft designs to be compared independently of the network routing. As expected, the smallest aircraft (plane A) has the largest per unit transportation cost, and the largest aircraft (plane C) has the smallest unit cost, which is due to the economies of scale captured by the cost models [4]. Further examination of Table 12 shows that although the unit transportation cost of the concurrent design aircraft (case 3 ) is slightly higher than the unit transportation cost of the vehicle design aircraft (case 2) and more aircraft are present in the case 3 architecture than in that of case 2, case 3 has a lower total system cost. This fact can be explained by the higher functional efficiency of the case 3 architecture.

The functional efficiency defines the percentage of used function in each case and is provided in Table 13 . The carrier capability use is defined as the ratio of the total cargo (package) weight being transported through the network $(2,284,006 \mathrm{lb})$ to the total capacity of each transportation architecture defined by each of the three optimization cases for the example network. The propulsive capability use is defined as the ratio of the total distance traveled in the network to the total range capability of all aircraft traveling in the network.

Examining Table 13 reveals that the vehicle design-only solution (case 2) has the highest capability use of all three cases, yet has the highest system cost. The concurrently optimized solution (case 3) has the highest capacity use of all three solutions and the lowest cost. Comparing this solution with cases 1 and 2 reveals that efficiency in carrier use is more important than efficiency in propulsive capability. This observation is supported by the dependence of design and operating costs on aircraft size and the assumption that the range of the aircraft is not significantly affected by the actual cargo loading. 
Table 12 Summary of results for three cases

\begin{tabular}{lccccc}
\hline \hline Parameter & Case 1: plane a & Case 1: plane b & Case 1: plane c & Case 2 & Case 3 \\
\hline Capacity $C, \mathrm{lb}$ & 5000 & 72,210 & 202,100 & 128,050 & 69,884 \\
Range $R, \mathrm{n}$ mile & 1063 & 3000 & 3950 & 1920 & 2560 \\
Fixed cost $f, \$ /$ day & 1481 & 10,616 & 26,129 & 14,106 & 9633 \\
Linear cost $m, \$ / \mathrm{h}$ & 758 & 3116 & 7194 & 4083 & 2807 \\
Unit cost, $\$ / \mathrm{lb} / \mathrm{n}$ mile & $3.7 \times 10^{-4}$ & $5.9 \times 10^{-5}$ & $4.2 \times 10^{-5}$ & $4.4 \times 10^{-5}$ & $5.0 \times 10^{-5}$ \\
Number used & 0 & 11 & 4 & 18 & 21 \\
\hline \hline
\end{tabular}

Table 13 Percent of use of aircraft capabilities for largest seven-city example

\begin{tabular}{lcc}
\hline \hline Case & Carrier capacity use & Propulsive capability use \\
\hline Case 1 & $75 \%$ & $40 \%$ \\
Case 2 & $50 \%$ & $61 \%$ \\
Case 3 & $78 \%$ & $47 \%$ \\
\hline \hline
\end{tabular}

\section{Conclusions}

In this paper, a methodology for integrated transportation network design was presented. By expanding the definition of a transportation system to include the vehicle definition as well as the network and operations during the design process, the system control volume was expanded to produce a system-level solution to the transportation architecture. Using the formulations developed to define the network, vehicle, and operations, a concurrent optimization of the transportation system definition is obtained for an example network in which a $10 \%$ improvement in cost over a traditional network analysis is realized.

Continuing work in this area centers around a relaxation of assumptions required to define the models used. For instance, the requirement that aircraft fly only a single round-trip route could be relaxed, allowing a single aircraft to visit multiple cities before returning to the original city; however, this expansion would require tracking the flight times to ensure feasible connections. The capacity and capability constraints were decoupled, by assuming that all aircraft operated at maximum capacity when evaluating aircraft capability. By including the package flows as part of the design vector, the actual capability of the vehicle could be assessed for a given route, but at the expense of computational complexity. Furthermore, the fidelity of the aircraft design models could be increased to a level that would be more effective at analyzing aircraft designs beyond the preconcept design phase.

This research could also be extended to analyze more complex problems. In the network models, the demand for cargo was assumed to be fixed; however, in reality, demand estimates are generally stochastic. This methodology could be extended to analyze probabilistic demand or analyze the effects of a demand evolution over time to define a robust transportation architecture. In the current design problem, only a single vehicle design was allowed. However, the problem formulation is defined such that extending the design problem to allow for multiple aircraft designs is straightforward. Such an analysis would provide a quantitative understanding of the appropriate fleet composition mixture and the effect of limiting the number of aircraft types allowed. An idealized solution might provide a customized aircraft for each route, which is clearly unrealistic in practice. In the case of multiple aircraft types, the analysis needs to reflect the requirements of operating a heterogeneous fleet, and therefore additional costs such as ground facility operations, maintenance, sparing, and cross training would need to be accounted for

The main innovation in this research lies in the problem decomposition and the embedded optimization formulation (Fig. 5 and Table 3 ) that uses an LP solver to ensure network flow feasibility within the nonlinear aircraft design problem. This methodology was developed to alleviate inefficiencies in the traditional simulated annealing framework and, along with the decomposition approach, provide good solutions to the integrated transportation system design problem in a reasonable time frame. Although the computational scalability of the embedded optimization framework for the

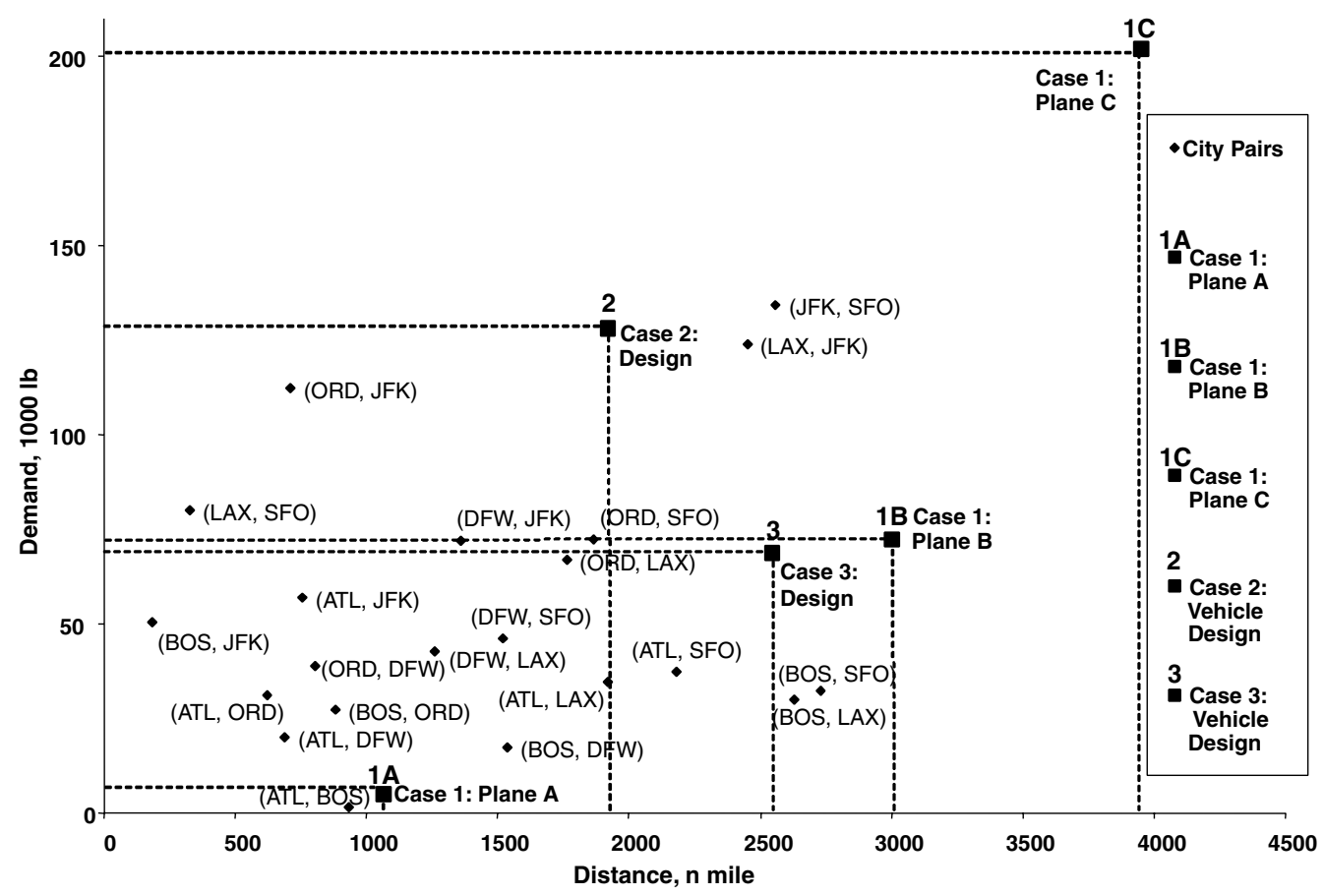

Fig. 9 Demand vs distance for the seven-city network; aircraft design envelopes (capacity and range) are shown superimposed as dashed boxes. 
integrated formulation in which larger city-pair networks with dozens or hundreds of nodes are examined remains to be investigated, initial investigations detailed by Taylor [21] show promise for this method.

The value of this analysis is not in the actual results obtained, but in the problem formulation. By expanding the definition of the system to include the vehicle, network, and operations, a more efficient system architecture can be obtained that reduces operating costs. This methodology can aid in strategic planning at a cargo or passenger airline by informing investment decisions, assisting with fleet planning, and understanding the commercial success or lack thereof of past designs. Alternatively, aircraft designers can use this methodology to customize variants and fine-tune specifications of future aircraft while considering the underlying network demand and route structure.

\section{References}

[1] Maier, M., "Architecting Principles for System-of-Systems," Systems Engineering, Vol. 1, No. 4, 1998, pp. 267-284

[2] Crossley, W., Mane, M., and Nusawardhana, "Variable Resource Allocation Using Multidisciplinary Optimization: Initial Investigations for System of Systems," 10th AIAA-ISSMO Multidisciplinary Analysis and Optimization Conference, AIAA Paper 2004-4605, 2004.

[3] Mane, M., Crossley, W., and Nusawarhana, "System of Systems Inspired Aircraft Sizing and Airline Resource Allocation via Decomposition," Journal of Aircraft (to be published).

[4] Raymer, D. P., Aircraft Design: A Conceptual Approach, 3rd ed., AIAA Education Series, AIAA, Washington, D.C., 1999.

[5] Barnhart, C., Boland, N., Clarke, L., Johnson, E., Nemhauser, G., and Shenoi, R., "Flight String Models for Aircraft Fleeting and Routing," Transportation Science, Vol. 32, No. 3, 1998.

[6] Ahuja, R., Magnanti, T., and Orlin, J., Network Flows: Theory, Algorithms and Applications, Prentice-Hall, Upper Saddle River, NJ, 1993.

[7] Dantzig, G., and Ramser, J., "The Truck Dispatching Problem," Management Science, Vol. 6, No. 1, 1959, pp. 80-91.

[8] Toth, P., and Vigo, D., (eds.), Vehicle Routing Problem, Monographs on Discrete Mathematics and Applications, Society for Industrial and
Applied Mathematics, Philadelphia, 2002.

[9] Simchi-Levi, D., Bramel, J., and Chen, X., The Logic of Logistics: Theory, Algorithms, and Applications for Logistics and Supply Chain Management, Springer, New York, 2005.

[10] Yang, L., and Kornfeld, R., "Examiniation of the Hub-and-Spoke Network: A Case Example Using Overnight Package Delivery," 41st Aerospace Sciences Meeting and Exhibit, AIAA Paper 2003-1334, 2003.

[11] Frommer, J., and Crossley, W., "Building Surrogate Models for Capability-Based Evaluation: Comparing Morphing and Fixed Geometry Aircraft in a Fleet Context," 6th AIAA Aviation Technology, Integration and Operations Conference, AIAA Paper 2006-7700, 2006.

[12] Meissinger, H., and Collins, J., "Mission Design and System Requirements for a Multiple-Function Orbital Transfer Vehicle," AIAA Space Technology Conference, AIAA Paper 99-42028, 1999.

[13] Wooster, P., Hofstetter, W., and Crawley, E., "Crew Exploration Vehicle Destination for Human Lunar Exploration: The Lunar Surface," Space 2005, AIAA Paper 2005-6626, 2005.

[14] Stanley, D., Cook, S., Connolly, J., and Hanley, J., "Exploration Systems Architecture Study: Overview of Architecture and Mission Operations Approach," SpaceOps 2006, AIAA Paper 2006-5935, 2006.

[15] Anderson, J., Aircraft Performance and Design, McGraw-Hill, New York, 1999.

[16] Bertsekas, D., Nonlinear Programming, Athena Scientific, Belmont, MA, 1999

[17] Bertsimas, D., and Weismantel, R., Optimization over Integers, Dynamic Ideas, Belmont, MA, 2004.

[18] Braun, R., and Kroo, I. M., "Development and Application of the Collaborative Optimization Architecture in a Multidisciplinary Design Environment," Multidisciplinary Design: State of the Art, edited by M. H. N. Alexandrov, Society for Industrial and Applied Mathematics, Philadelphia, 1997, pp. 98-116.

[19] Sobieszczanski-Sobieski, J., "Integrated System-of-System Synthesis (ISSS)," 11th AIAA/ISSMO Multidisciplinary Analysis and Optimization Conference, AIAA 2006-7064, 2006.

[20] Kirkpatrick, S., Gelatt, C. D., and Vecchi, M. P., "Optimization by Simulated Annealing," Science, Vol. 220, No. 4598, 1983, pp. 671680.

[21] Taylor, C., Integrated Transportation System Design Optimization, Ph.D. Thesis, Massachusetts Inst. of Technology, Cambridge, MA, Jan. 2007. 